\title{
THE VERY LONG BASELINE ARRAY
}

\author{
P. J. NAPIER \\ National Radio Astronomy Observatory*, Socorro, New Mexico, USA
}

January, 1993

\begin{abstract}
.
The VLBA is a new radiotelescope dedicated to research in astronomy and astrometry/geodesy using the techniques of VLBI. The array comprises ten $25 \mathrm{~m}$ diameter reflector antennas located so as to give optimum coverage of baselines up to $8600 \mathrm{~km}$. Instrumentation provided at each antenna includes receivers for nine frequency bands between $327 \mathrm{MHz}$ and $43 \mathrm{GHz}$, a hydrogen maser frequency standard and a tape recorder system capable of a peak data rate of $512 \mathrm{Mb} / \mathrm{s}$ and unattended operation for a day recording at $128 \mathrm{Mb} / \mathrm{s}$. The antennas are controlled and monitored by a single operator located in the Array Operations Center (AOC) in Socorro, New Mexico. The AOC also houses the 20 station correlator which processes the data recorded at the antennas.
\end{abstract}

Key words: VLBA - VLBI

\section{Introduction}

The technique of Very Long Baseline Interferometry (VLBI), in which radio interferometry is performed using data recorded on wide-bandwidth tape recorders at widely separated antennas equipped with stable clocks, has been in use for more than 25 years (1). VLBI techniques have now matured sufficiently (2) so that high resolution observations of astronomical objects are regularly scheduled using arrays composed of antennas provided by numerous radio observatories around the world. The data provided by such ad hoc arrays suffer from several limitations including limited observing time, non-optimum location of antennas, inadequate common frequency coverage between array elements and data calibration problems resulting from non-identical antennas and receiving systems. The Very Long Baseline Array (VLBA) is a new instrument dedicated to VLBI research in astronomy and astrometry/geodesy and is designed to overcome many of these limitations. It is scheduled to begin full operation in 1993 .

Many aspects of the VLBA have been described elsewhere, including a general description and its principles of operation $(3 ; 4)$, a summary of instrumental parameters $(5 ; 6)$, the optimized array layout $(7)$, the wide-bandwidth tape recorders (8), the phase calibration system (9), the correlator (10), and details of the array layout, antennas and all instrumentation (11). In this paper we will briefly review the principal design goals of the VLBA and comment on the degree to which early tests and observations show that they have been met.

\section{VLBA Design Goals}

We will discuss the design goals for the VLBA under eight headings (taken from (11)).

* The National Radio Astronomy Observatory is operated by Associated Universities Inc. under a cooperative agreement with the National Science Foundation 


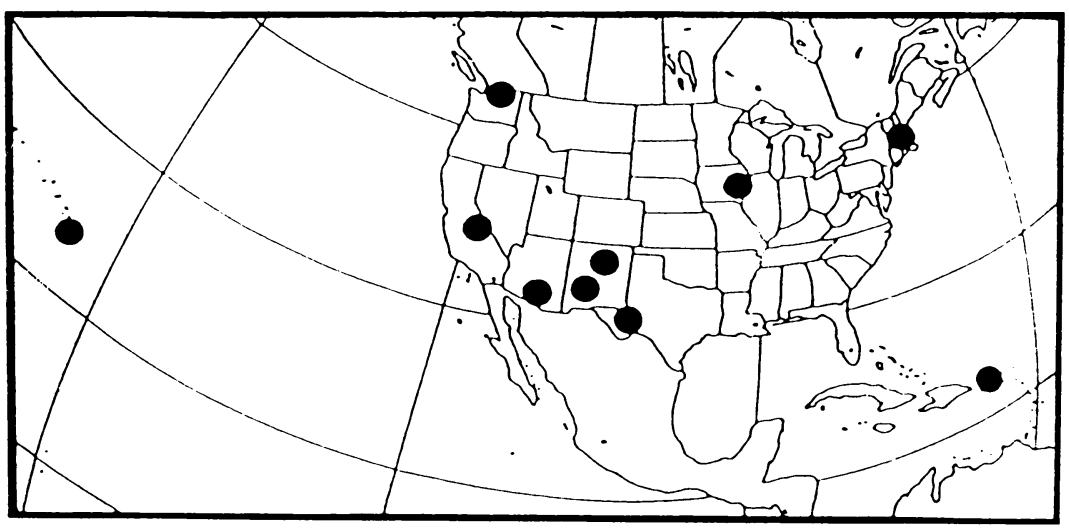

Fig. 1. VLBA array configuration.

\subsection{High Image Quality}

The VLBA is designed to provide high resolution images with high dynamic range. Image resolution is determined by the length of the longest baseline in the array and this was chosen to be the longest baseline available on conveniently accessible US Territory. The longest baseline, $8600 \mathrm{~km}$, is between the most easterly array element on the island of St. Croix, US Virgin Islands, and the most westerly element on Mt. Mauna Kea on the island of Hawaii. The dynamic range of images produced by a synthesis array is determined by how well the array samples the $(\mathrm{u}, \mathrm{v})$ plane (spatial frequency plant) and by the calibration quality of the measured visibility data. The numLer of VLBA array elements was chosen, primarily for budgetary reasons, to be ten. These elements are arranged as shown in Figure 1, and listed in Table I, so as to give optimum sampling of the $8600 \mathrm{~km}$ aperture. The $(\mathrm{u}, \mathrm{v})$ plane coverage provided

\section{TABLE I}

Locations of the VLBA Antennas.

\begin{tabular}{lllll}
\hline Code & Location & $\begin{array}{l}\text { N Latitude } \\
\text { (d.m.s) }\end{array}$ & $\begin{array}{l}\text { W Longitude } \\
\text { (d.m.s) }\end{array}$ & $\begin{array}{l}\text { Elevation } \\
(\mathrm{m})\end{array}$ \\
\hline SC & St. Croix VI & 174530.57 & 643502.61 & 16 \\
HN & Hancock NH & 425600.96 & 715911.69 & 309 \\
NL & N. Liberty IA & 414617.03 & 913426.35 & 241 \\
FD & Fort Davis TX & 303805.63 & 1035639.13 & 1615 \\
LA & Los Alamos NM & 354630.33 & 1061442.01 & 1967 \\
PT & Pie Town NM & 341803.61 & 1080707.24 & 2371 \\
KP & Kitt Peak AZ & 315722.39 & 1113642.26 & 1916 \\
OV & Owens Valley CA & 371354.19 & 1181633.98 & 1207 \\
BR & Brewster WA & 480752.80 & 1194055.34 & 255 \\
MK & Mauna Kea HI & 194815.85 & 1552728.95 & 3720 \\
\hline
\end{tabular}




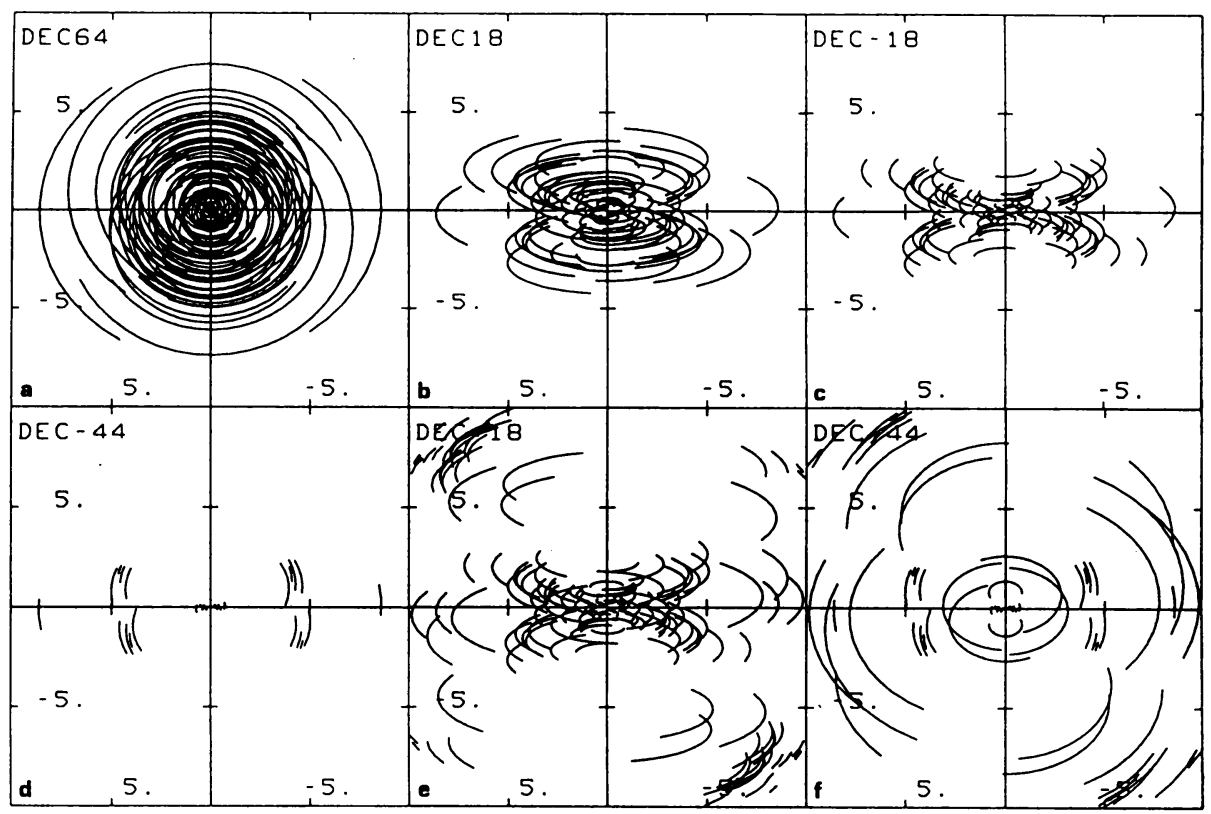

Fig. 2. (a)-(d) (u,v) plane coverage for the VLBA for declinations $64^{\circ}, 18^{\circ},-18^{\circ},-44^{\circ}$. (e),(f) VLBA plus Narrabri, Hobart, Perth, Hartebeesthoek for declinations $-18^{\circ}$ and $-44^{\circ}$.

by this array is shown in Figure 2. The coverage is good for northern declinations but is clearly quite inadequate in the south. As an example of the improvement that is possible with the addition of a few antennas located in southern latitudes, Figure 2 also shows the coverage provided by an array composed of the VLBA and four southern hemisphere antennas. The southern VLBI antennas used in the example are three in Australia, at Narrabri (the Australia Telescope), Hobart and Perth and one in South Africa at Hartebeesthoek. Note that the assumption is made in Figure 2 that all antennas track down to a lower elevation limit of $10^{\circ}$. The VLBA antennas can, in general, observe down to $2^{\circ}$. The resolution provided by the 8600 $\mathrm{km}$ VLBA aperture, for the various frequency bands supported by VLBA receivers, is shown in Table II.

Several measures have been taken to ensure that the VLBA produces well calibrated data. Since the antennas are nominally identical it has been possible to measure, understand and calibrate the pointing and gain-vs-elevation curves of all antennas at all frequency bands with much less effort than would be required for dissimilar antennas. Both the pointing ( 8 arcsec rms per axis) and the gain-vs-elevation curve are acceptable for operation at $43 \mathrm{GHz}$. The total system temperature of the receiver systems is continuously monitored using a low level switched noise calibration signal injected at the input of the low-noise receivers. Tests show that these measures produce visibility data with amplitudes accurate to a few $\%$ for the centimeter wavelength bands. Phase calibration of the visibility data is improved using 
the active phase measurement systems mentioned in Section 2.5 below. Finally, care has been taken in the design of the electronics system to minimize effects that cause instrumental closure errors so that self-calibration techniques will work well.

Early demonstrations of the ability of the VLBA to produce good quality data are the $43 \mathrm{GHz}$ spectral line studies of $\mathrm{SiO}$ masers and the image of the jet in Mrk 501 reported in (12). The image of Mrk 501 was made with only 4 VLBA antennas and 1 VLA antenna, but has a dynamic range of 2400 and took far fewer cycles of self-calibration than is usual for an image of this quality.

\subsection{High Sensitivity}

The VLBA has the highest sensitivity possible within the constraints of the construction and operating budgets. The construction budget limited the antenna diameter to $25 \mathrm{~m}$ and the desire for reliable, low cost operation led to the use of cryogenically cooled transistor amplifiers for the low noise amplifiers in the receivers. The amplifiers for the two lowest frequency bands are not cooled. The measured sensitivities (taken from (11)) for the VLBA antennas in the nine frequency bands are shown in Table II. Also shown is the calculated (from (13)) sensitivity for a single VLBA baseline for a 2 min integration time and standard continuum bandwidth of $64 \mathrm{MHz}(128 \mathrm{Mb} / \mathrm{sec}$ data rate). The. number given is the $1 \sigma$ noise in either the real or imaginary component of the visibility measured on 1 baseline with 1 polarization.

TABLE II

VLBA Frequency Bands.

\begin{tabular}{|c|c|c|c|c|c|}
\hline $\begin{array}{l}\text { Band } \\
\text { Name } \\
(\mathrm{cm})\end{array}$ & $\begin{array}{l}\text { Nominal } \\
\text { Band } \\
(\mathrm{GHz})\end{array}$ & $\begin{array}{l}\text { Tunable } \\
\text { Band } \\
(\mathrm{GHz})\end{array}$ & $\begin{array}{l}\text { Measured } \\
\text { System } \\
\text { Temperature } \\
(\mathrm{Jy})\end{array}$ & $\begin{array}{l}1 \text { baseline } \\
\text { sensitivity } \\
(1 \sigma) \\
(\mathrm{mJy})\end{array}$ & Resolution \\
\hline 90 & $.312-.342$ & $.305-.350$ & 1930 & 28 & 22 \\
\hline 50 & $.595-.625$ & $.590-.630$ & 1950 & 29 & 12 \\
\hline 20 & $1.35-1.75$ & $.2-1.85$ & 300 & 4 & 5 \\
\hline 13 & $2.15-2.35$ & $2.0-2.8$ & 330 & 5 & 3 \\
\hline 6 & $4.6-5.1$ & $4.4-5.2$ & 275 & 4 & 1.4 \\
\hline 4 & $8.0-8.8$ & $7.8-9.1$ & 290 & 4 & 0.9 \\
\hline 2 & $12.1-15.4$ & $11.8-15.7$ & 400 & 6 & 0.5 \\
\hline 1.3 & $21.7-24.1$ & $21.1-25.1$ & 800 & 12 & 0.3 \\
\hline 0.7 & $41-45$ & $40-46$ & 1100 & 16 & 0.2 \\
\hline
\end{tabular}

The VLBA antennas have been designed with reasonably high slew rates $\left(30^{\circ} / \mathrm{min}\right.$ in elevation, $90^{\circ} / \mathrm{min}$ in azimuth) so that they can quickly switch between pairs of sources. This allows phase referencing to be used to extend integration time, as demonstrated in (14).

Sensitivity can also be increased by increasing the recorded data rate. A single VLBA tape recorder can record at a maximum rate of $256 \mathrm{Mb} / \mathrm{sec}$ and if both of 
the recorders provided at each site are operated together at maximum rate, 512 $\mathrm{Mb} / \mathrm{sec}$ is possible.

\subsection{Adequate Frequency Coverage}

The VLBA antennas are equipped with feeds and receivers to sample the frequency range $300 \mathrm{MHz}$ to $43 \mathrm{GHz}$ in nine bands, as shown in Table II. In Table II the measured system temperature is measured at the VLBI Network frequency and is typical of the performance of the most sensitive antennas in the nominal band. The tunable band is the frequency range over which observations can be made, but with a loss of sensitivity of about a factor of two compared with the nominal band.

The antennas have been designed to have useful performance at $86 \mathrm{GHz}$ and tests on the first antenna gave a measured aperture efficiency of $18 \%$ at this frequency. A location for an $86 \mathrm{GHz}$ receiver, as well as an additional unused receiver location, are available in the receiver room for future use. All receivers are dual circularly polarized and can be selected under computer control in less than 20 sec.

\subsection{Spectral Line Imaging}

The VLBA correlator provides adequate spectral resolution and spectral dynamic range to allow the study of astronomical masers. Each station input to the correlator comprises up to 8 channels, each of which can have a bandwidth of $0.0625,0.125$, $0.25,0.5,1,2,4,8$, or $16 \mathrm{MHz}$. Each input channel can be resolved into 1024, $512,256,128,64$, or 32 spectral points. When both polarizations are correlated to produce all four Stokes parameters, the maximum number of spectral points is 512 . The shortest integration time for the correlator is $131.072 \mathrm{msec}$. Pulsar gating is available in the correlator and the 20 input stations can be subdivided into any number of subarrays, with the smallest allowable array size being a single antenna, to allow parallel processing of several experiments.

\subsection{GeOdesy/ASTROMETRY RESEARCH}

As well as being an imaging instrument, the VLBA is designed to support research in geodesy and astrometry. This requires the precise measurement of the time delays at each antenna. A dual frequency simultaneous observing mode for $2.3 / 8.4 \mathrm{GHz}$ is provided to allow for the calibration of ionospheric delays. In this mode the sensitivity at $8.4 \mathrm{GHz}$ is approximately $20 \%$ worse than the value given in Table II. The instrumental path lengths in each receiving system are carefully controlled and monitored. At each site a measurement system continuously monitors the length of the signal path used to distribute reference signals from the hydrogen maser frequency standard, located in a building near the antenna, to the antenna. Another measurement system, "the pulse calibration system", continuously monitors the length of the signal path from the receiver input to the signal digitizer. During its commissioning phase the VLBA has successfully participated in a number of geodetic experiments as part of the Crustal Dynamics Project. 


\subsection{USER FRIENDLY}

A significant goal for the VLBA is that it will be sufficiently "user friendly" so that it can be used by researchers who are not experts in interferometric techniques. The array is scheduled and operated as a unified instrument from the NRAO Array Operations Center (AOC) in Socorro, New Mexico. A single operator in the AOC continuously monitors and controls the array in accordance with simple source files prepared in advance by the scheduled observer. Tapes will be returned to the correlator in the AOC where correlation is performed under the control of a correlator operator. First time observers will be encouraged to come to the AOC for post processing of their correlated data which will typically be carried out using the NRAO Astronomical Image Processing (AIPS) software package.

\subsection{Full Time Operation}

The VLBA will be operated continuously except for scheduled weekly maintenance periods. In order to reduce operating costs typically only two technicians are employed at each of the antennas. All equipment is highly automated and operates unattended most of the time. Recording tapes will be changed once a day and all equipment is modularized so that, in the event of a failure, repairs can be made by simply installing a replacement module shipped to the site from the AOC. It is anticipated that the VLBA will be dynamically scheduled to take advantage of good weather for high frequency observations, or to ameliorate the effect of a piece of failed equipment at an antenna, or to respond to a "target of opportunity". An early example of the latter is the VLBA observations of the flare in the variable star HR1099 reported in (12). For these observations the 6 available VLBA antennas started observing in Mk IIIA recording mode 3 hours after AOC personnel received word of the flare.

\subsection{Operation With non-VlBa antennas}

As well as operating as a stand-alone instrument the VLBA is scheduled for a significant fraction of the time to operate in conjunction with other VLBI telescopes. This allows improved $(u, v)$ plane coverage, for example the Southern Hemisphere case given in section 2.1 above, or improved sensitivity resulting from the inclusion of the largest single dish apertures. Another example of improved (u,v) coverage is shown in Figure 3 which shows the improved coverage which can be obtained for northern declinations with the addition of only four European telescopes. The VLBA correlator is capable of processing the inputs from 20 stations simultaneously and can read tapes written in the widely used Mk IIIA format, providing compatibility with more than 25 antennas around the world.

\section{Conclusion}

The VLBA is a highly flexible instrument for high resolution research in radio astronomy. It is expected that it will make VLBI techniques available to researchers 


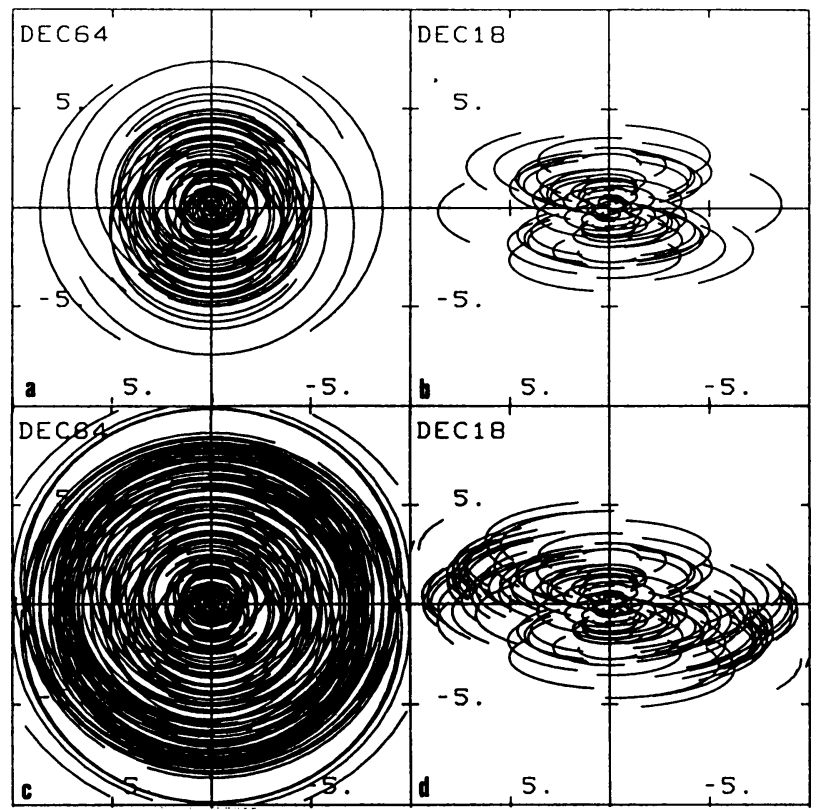

Fig. 3. (a),(b). (u,v) plane coverage for the VLBA for declinations $64^{\circ}$ and $18^{\circ}$. (c),(d) VLBA plus Bonn, Jodrell, Noto and Onsala.

who are not experts in interferometric techniques. Proposals to use the VLBA should be addressed to the NRAO Director.

\section{References}

[1] Klemperer, W.K.: 1972, "Long Baseline Radio Interferometry with Independent Frequency Standards", Proc. IEEE, 60, 602-609.

[2] Thompson A.R., Moran, J.M and Swenson, G.W: 1986, Interferometry and Synthesis in Radio Astronomy, John Wiley and Sons Pub., New York, reprinted by Krieger Press, Melbourne, Fl, 1991.

[3] Kellerman, K.I. and Thompson, A.R.: 1985, "The Very Long Baseline Array",Science, 229, 123-130.

[4] Kellerman, K.I. and Thompson, A.R.: 1988, "The Very Long Baseline Array", Scientific American, 258, 1, 54-63.

[5] Napier, P.J.: 1991, "The Very Long Baseline Array", Radio Interferometry: Theory, Techniques and Applications, IAU Coll. 191, ASP Conf. Series, 19, 390-394.

[6] Napier, P.J.: 1992, "The Large Synthesis Radio Telescopes of the National Radio Astronomy Observatory", IEEE-MTT Internat. Microwave Symp. Digest, 3, 1243-1246.

[7] Walker, R.C.: 1983, "VLBI Array Design",Proc. Internat. Symp. on Indirect Imaging, Sydney, Australia, Cambridge University Press, 53-65.

[8] Hinteregger, H.F., Rogers, A.E.E., Cappallo, R.C., Webber, J.C., Petrachenko, W.T. and Allen, H.: 1991, "A High Data Rate Recorder for Astronomy", IEEE Trans. on Magnetics, MAG-27, 3455-3465.

[9] Rogers, A.E.E., Corey, B.E., Nesman, E.F.: 1992, "Phase Calibration of the VLBA", IEEEMTT Internat. Microwave Symp. Digest, 3, 1373. 
[10] Romney, J.D., Benson, J.M., Broadwell, C.M., Escoffier, R.P., Gonzalez, R.D., Greenberg, J.H., Horstkotte, J.E., Runion, M.E. and Wells, D.C.: 1993, "The VLBA Correlator", in preparation.

[11] Napier, P.J., Bagri, D.S., Clark, B.G., Rogers, A.E.E., Romney, J.D., Thompson, A.R., Walker, R.C.: 1993, "The Very Long Baseline Array", submitted to Proc. IEEE, Special Issue on the Design and Instrumentation of Antennas for Deep Space Telecommunications and Radioastronomy, November, 1993.

[12] Diamond, P.J. and Beasley, A.J.: "Recent Results from the VLBA", these Proceedings.

[13] Walker, R.C.: 1989, "Sensitivity", in Very Long Baseline Interferometry, Techniques and Applications, M. Felli and R. E. Spencer editors, Kluwer Academic Publishers, Dordrecht, 163-182.

[14] Beasley, A.J., Conway, J.E., Diamond, P.J., Cotton, W.D. and Vermeulen, R.C.: "VLBA Phase Referencing", these Proceedings.

\section{Discussion:}

Davies:

It is important that we make best use of a combination of the VLBA with other arrays such as the European VLBI Network (EVN). We need to put in hand arrangements to achieve this goal; discussions are still at an early stage. Can you comment? Also, could you indicate the role the Green Bank Telescope will play in VLBI?

Napier:

It is NRAO's intention at this time to continue the practice of past years, ie to participate in four sessions per year, each approximately 3 weeks in length, during which observations will be coordinated with the EVN. The GBT electronics design is fully compatible with coordinated observing with the VLBA and a VLBA Data Acquisition System will be provided. The GBT will be available for VLBI observations and the amount of time will be determined by how competetive the VLBI science is with the single dish science. 